\title{
Iterated towers of number fields by a quadratic map defined over the Gaussian rationals
}

\author{
By Yasushi Mizusawa*) and Kota Yamamoto**)
}

(Communicated by Shigefumi MoRI, M.J.A., Sept. 14, 2020)

\begin{abstract}
An iterated tower of number fields is constructed by adding preimages of a base point by iterations of a rational map. A certain basic quadratic rational map defined over the Gaussian number field yields such a tower of which any two steps are relative bicyclic biquadratic extensions. Regarding such towers as analogues of $\mathbf{Z}_{2}$-extensions, we examine the parity of 2ideal class numbers along the towers with some examples.
\end{abstract}

Key words: Iterated extension; class number parity; Iwasawa theory.

1. Introduction. Let $\phi$ be a rational map of prime degree $p$ which is defined as the rational function $\phi(x) \in k(x)$ over a number field $k$. Let $\left\{b_{n}\right\}_{0 \leq n \in \mathbf{Z}}$ be a sequence of algebraic numbers such that $\bar{\phi}\left(b_{n+1}\right)=b_{n}$ for all $n \geq 0$. For a finite extension $K / k\left(b_{0}\right)$, we obtain a sequence

$$
K \subset K_{1} \subset K_{2} \subset \cdots \subset K_{n} \subset \cdots \subset K_{\infty}=\bigcup_{n \geq 1} K_{n},
$$

where $K_{n}=K\left(b_{n}\right)$ for each $n \geq 1$. The number field $K_{n}$ is contained in a Galois extension $K\left(\phi^{-n}\left(b_{0}\right)\right)$ of $K$. If $\phi$ is 'post-critically finite', i.e., the orbits $\left\{\phi^{n}(c)\right\}_{0 \leq n \in \mathbf{Z}}$ of any critical points $c$ of $\phi$ are finite, then the number of primes of $K$ ramifying in $K\left(\phi^{-\infty}\left(b_{0}\right)\right) / K$ is finite $([1,5])$, and such iterated extensions have been constructed and studied in various situations (see e.g. [2,3]).

If $K_{n} / K$ is a cyclic Galois extension of degree $p^{n}$ for all $n \geq 1$, then $K_{\infty}$ is a $\mathbf{Z}_{p}$-extension of $K$, i.e., $\operatorname{Gal}\left(K_{\infty} / K\right)$ is isomorphic to the additive group $\mathbf{Z}_{p}$ of $p$-adic integers. For example, $K_{\infty} / K$ is the cyclotomic $\mathbf{Z}_{2}$-extension if $\phi(x)=x^{2}-2$ and $b_{0}=0$. The growth of the $p$-parts of the class numbers along a $\mathbf{Z}_{p}$-extension $K_{\infty} / K$ is described by Iwasawa's class number formula ([9]), and such a formula has been extended to some non-Galois towers $([4,11]$ etc.) and $p$-adic Lie extensions $([6,12,15]$ etc.). One of the most important con-

2020 Mathematics Subject Classification. Primary 11R11; Secondary 11R29, 11R23.

*) Department of Mathematics, Nagoya Institute of Technology, Gokiso-cho, Showa-ku, Nagoya 466-8555, Japan.

**) Division of Mathematics and Mathematical Science, Department of Computer Science and Engineering, Graduate School of Engineering, Nagoya Institute of Technology, Gokisocho, Showa-ku, Nagoya 466-8555, Japan. jectures in Iwasawa theory is Greenberg's conjecture $([7])$ which states that the $p$-parts of the class numbers are bounded along the cyclotomic $\mathbf{Z}_{p}$-extension of a totally real number field. Analogous problems can be also considered for iterated extensions $K_{\infty} / K$ by post-critically finite $\phi$, as in [17] where the case of $\phi(x)=x^{2}-2$ has been considered. In particular, it is a basic problem to find many iterated extensions $K_{\infty} / K$ such that the $T$-ideal class number of $K_{n}$ is not divisible by $p$ for all sufficiently large $n$ and for a finite set $T$ of primes of $K$ ramifying in $K_{\infty} / K$. For a finite set $T$ of primes of a subfield of a number field $F$, the $T$-ideal class number $\left|C l^{T}(F)\right|$ of $F$ is the order of the $T$-ideal class group $C l^{T}(F)=C l(F) /\left\langle[w] ; w \mid \prod_{v \in T} v\right\rangle$, which is the quotient of the ideal class group $C l(F)$ by the subgroup generated by all classes of prime ideals $w$ lying over $T$.

In this paper, we consider iterated extensions $K_{\infty} / K$ by a rational finction

$$
\phi(x)=\frac{(i x)+(i x)^{-1}}{2}=\frac{i}{2}\left(x-\frac{1}{x}\right)
$$

of degree $p=2$, which is defined over the Gaussian number field $\mathbf{Q}(i)$, where $i=\sqrt{-1}$. The map $\phi$ is post-critically finite, and comes from an endomorphism of the elliptic curve $E: y^{2}=x^{3}+x$ with complex multiplication by Gaussian integers $\mathbf{Z}[i]$ (see Remark 3.3). Then, regarding $K_{\infty} / K$ as an analogue of a $\mathbf{Z}_{2}$-extension, we examine the parity of $\left|C l^{T}\left(K_{n}\right)\right|$ along the tower of iterated extensions $\left\{K_{n}\right\}_{1 \leq n \in \mathbf{Z}}$. The main result is the following theorem, which can be seen as a partial refinement of $[5, \S 5]$ in a special case. Put a condition $C(b)$ : 


$$
\left\{\sqrt{b^{2}-1}, \sqrt{b(b \pm 1)}, \sqrt{b \pm 1}, \sqrt{b}\right\} \cap k(b)=\emptyset
$$

for each algebraic number $b$. We denote an abelian 2 -group by its type.

Theorem 1.1. Suppose that $i \in k$ and $\phi(x)=\frac{i}{2}\left(x-\frac{1}{x}\right)$. Let $b_{0} \in k$ be an algebraic integer satisfying $C\left(b_{0}\right)$. Put $k_{n}=k\left(b_{n}\right)$ for $n \geq 0$. Put $K=$ $k\left(\sqrt{b_{0}}\right)$, and put $K_{n}=k_{n}\left(\sqrt{b_{0}}\right)$ for $n \geq 1$. Let $T$ be $a$ set of primes of $k$ lying over 2 . Then the following statements hold true for each $n \geq 1$.

(a) $k_{n+1} / k_{n-1}$ is a $[2,2]$-extension.

(b) $K_{n+3} / K_{n}$ is a $[2,4]$-extension unramifid outside 2.

(c) If $K_{n+2} / K_{n}$ is totally ramified at any prime lying over 2 and $2 \nmid\left|C l^{T}\left(K_{n+2}\right)\right|$, then $2 \nmid\left|C l^{T}\left(K_{m}\right)\right|$ for all $m \geq n$.

In the proof of Theorem 1.1, the notation $K_{0}$ denotes either $k$ or $K$ according to whether $\sqrt{b_{0}\left(b_{0}^{2}-1\right)} \in k$ or not. Then we will see that the statements (b) and (c) also hold true for $n=0$ if $\sqrt{b_{0}\left(b_{0}^{2}-1\right)} \in k$.

Remark 1.2. For $b_{0} \in k$, the conditions $C\left(b_{0}\right)$ and $\sqrt{b_{0}\left(b_{0}^{2}-1\right)} \notin k$ are satisfied if and only if $k\left(\sqrt{b_{0}}, \sqrt{b_{0}+1}, \sqrt{b_{0}-1}\right) / k$ is a $[2,2,2]$-extension. If $\sqrt{b_{0}\left(b_{0}^{2}-1\right)} \in k$, then the condition $C\left(b_{0}\right)$ is satisfied if and only if $k\left(\sqrt{b_{0}}, \sqrt{b_{0}+1}\right) / k$ is a $[2,2]$-extension. The point $\left(b_{0}, \sqrt{b_{0}\left(b_{0}^{2}-1\right)}\right)$ is a $k\left(\sqrt{b_{0}\left(b_{0}^{2}-1\right)}\right)$-rational point of the elliptic curve $E^{\prime}: y^{2}=x^{3}-x$.

\section{Proof of Theorem 1.1.}

2.1. Preliminaries. Suppose that $\phi$ is defined as (1), and $k$ is a finite extension of $\mathbf{Q}(i)$. For $b \in \overline{\mathbf{Q}}$, we have $\phi\left(b^{\prime}\right)=b$ if and only if $b^{2}+2 b i b^{\prime}-1=0$, i.e.,

$$
b^{\prime}=-i\left(b \pm \sqrt{b^{2}-1}\right)=\left(\frac{\sqrt{b-1} \pm \sqrt{b+1}}{1+i}\right)^{2} .
$$

Then $k\left(b^{\prime}\right)=k\left(b, \sqrt{b^{2}-1}\right)$. Note that $2 i=(1+i)^{2}$.

Lemma 2.1. Assume that $i \in k$ and that the condition $C(b)$ is satisfied for $b \in \overline{\mathbf{Q}}$. Let $b^{\prime}$ and $b^{\prime \prime}$ be algebraic numbers such that $\phi\left(b^{\prime}\right)=b$ and $\phi\left(b^{\prime \prime}\right)=$ $b^{\prime}$. Then $k\left(b^{\prime \prime}\right) / k(b)$ is a $[2,2]$-extension containing three quadratic subextensions $k(b, \sqrt{b(b \pm 1)}), k\left(b^{\prime}\right)$. Moreover, the condition $C\left(b^{\prime}\right)$ is also satisfied.

Proof. Let

$$
f(x)=x^{4}+4 b x^{3}+2 x^{2}-4 b x+1 \in k(b)[x]
$$

be the numerator of $\phi^{2}(x)-b=\frac{f(x)}{-4 x^{3}+4 x}$. Note that $\phi\left(-\frac{1}{x}\right)=\phi(x)$. Since $\phi\left(b^{\prime \prime}\right)=b^{\prime}$ and $\phi\left(b^{\prime}\right)=b$, we can easily see that the four roots of $f(x)$ are

$$
b^{\prime \prime}, \quad-\frac{1}{b^{\prime \prime}}, \quad \frac{b^{\prime \prime}+1}{b^{\prime \prime}-1}, \quad-\frac{b^{\prime \prime}-1}{b^{\prime \prime}+1},
$$

and hence $k\left(b^{\prime \prime}\right) / k(b)$ is a Galois extension and $\left[k\left(b^{\prime \prime}\right): k(b)\right] \leq 4$. Moreover, one can directly show that

$$
\left(b^{\prime \prime}+\frac{b^{\prime \prime}+1}{b^{\prime \prime}-1}+2 b\right)^{2}=4 b(b-1),
$$

which implies that $\sqrt{b(b-1)} \in k\left(b^{\prime \prime}\right)$. Since the condition $C(b)$ is satisfied,

$$
k\left(b^{\prime \prime}\right)=k(b)(\sqrt{b(b-1)}, \sqrt{b(b+1)})
$$

and $\operatorname{Gal}\left(k\left(b^{\prime \prime}\right) / k(b)\right) \simeq[2,2]$. Thus we obtain the former statement.

Since $\left[k\left(b^{\prime \prime}\right): k\left(b^{\prime}\right)\right]=2$, we have $\sqrt{b^{\prime 2}-1} \notin$ $k\left(b^{\prime}\right)$. Recall that $b^{\prime 2}+2 b i b^{\prime}-1=0$. Since $N_{k\left(b^{\prime}\right) / k(b)}\left(b^{\prime}\left(b^{\prime} \pm 1\right)\right)=-N_{k\left(b^{\prime}\right) / k(b)}\left(b^{\prime} \pm 1\right)= \pm(1+i)^{2} b$, and since $\sqrt{b} \notin k(b)$ by the assumption, we have $\sqrt{b^{\prime}\left(b^{\prime} \pm 1\right)} \notin k\left(b^{\prime}\right)$ and $\sqrt{b^{\prime} \pm 1} \notin k\left(b^{\prime}\right)$. Note that $k\left(b^{\prime \prime}\right)=k\left(b^{\prime}\right)\left(\sqrt{b^{\prime 2}-1}\right)=k\left(b^{\prime}\right)\left(\sqrt{b b^{\prime}}\right)$. If $\sqrt{b^{\prime}} \in k\left(b^{\prime}\right)$, then $k\left(b^{\prime \prime}\right)=k\left(b^{\prime}\right)(\sqrt{b})$, in particular $k(\sqrt{b}) / k(b)$ is a quadratic subextension of the $[2,2]$-extension $k\left(b^{\prime \prime}\right) / k(b)$. This implies that $k(\sqrt{b})=$ $k(b, \sqrt{b(b-1)})$ or $\quad k(\sqrt{b})=k(b, \sqrt{b(b+1)})$, i.e., $\sqrt{b-1} \in k(b)$ or $\sqrt{b+1} \in k(b)$. This is a contradiction. Therefore $\sqrt{b^{\prime}} \notin k\left(b^{\prime}\right)$. Thus the latter statement is also obtained.

Remark 2.2. Since $\phi^{2}$ is defined over $\mathbf{Q}$, $f(x)$ is defined over $\mathbf{Q}(b)$. Put $g(x)= \pm \sqrt{x}$. Then $g^{-1}(x)=x^{2}$, and a conjugate

$$
\left(g^{-1} \phi g\right)(x)=-\frac{1}{4}\left(x-2+\frac{1}{x}\right)
$$

is also defined over $\mathbf{Q}$.

2.2. Field theoretic part. Unless otherwise noted, we may suppose that $b_{0} \in k$ is not necessarily an algebraic integer.

Lemma 2.3. If $C\left(b_{0}\right)$ is satisfied, then for all $n \geq 0, C\left(b_{n}\right)$ is satisfied, and $k_{n+2} / k_{n}$ is a [2,2]extension containing three quadratic subextensions $k_{n}\left(\sqrt{b_{n}\left(b_{n} \pm 1\right)}\right), k_{n+1}=k_{n}\left(\sqrt{b_{n}^{2}-1}\right)$.

Proof. We obtain the statement by using Lemma 2.1 inductively.

Throughout the following, we assume that $C\left(b_{0}\right)$ is satisfied. Recall that $K_{n}=k_{n}\left(\sqrt{b_{0}}\right)$ for each $n \geq 1$, and put

$$
\begin{aligned}
N_{0} & =\left\{1 \leq n \in \mathbf{Z} \mid \sqrt{b_{0}} \in k_{n}\right\} \\
& =\left\{1 \leq n \in \mathbf{Z} \mid K_{n}=k_{n}\right\} .
\end{aligned}
$$


Note that $n \in N_{0}$ if $n \geq \min N_{0}$.

Lemma 2.4. If $n \geq 1$, then $K_{n+1}=K_{n}\left(\sqrt{b_{n}}\right)$. In particular, $K_{n}=k_{n}$ and $k_{n+1}=k_{n}\left(\sqrt{b_{n}}\right)$ for all $n \in N_{0}$.

Proof. Recall that $k_{n+1}=k_{n}\left(\sqrt{b_{n-1} b_{n}}\right)$ for all $n \geq 1$. Then $K_{n+1}=K_{n}\left(\sqrt{b_{n-1} b_{n}}\right)$. If $\sqrt{b_{n-1}} \in K_{n}$ for $n \geq 1$, then $K_{n+1}=K_{n}\left(\sqrt{b_{n}}\right)$ and $\sqrt{b_{n}} \in K_{n+1}$. Since $\sqrt{b_{0}} \in K_{1}$, we obtain the claim by induction.

Lemma 2.5. $K_{n+3} / k_{n}$ is a Galois extension for each $n \geq 0$.

Proof. Recall that $-\frac{1}{b_{n+1}}$ is the conjugate of $b_{n+1}$ over $k_{n}$. Then there exists $\tau \in \operatorname{Gal}\left(\overline{\mathbf{Q}} / k_{n}\right)$ such that $b_{n+1}^{\tau}=-\frac{1}{b_{n+1}}$. Since $k_{n+3} / k_{n+1}$ is a Galois extension, the conjugate of $k_{n+3}$ over $k_{n}$ different from $k_{n+3}$ itself is $k_{n+3}^{\tau}=k\left(b_{n+3}^{\tau}\right)$. Therefore $k_{n+3}^{\tau} k_{n+3}=k_{n+3}\left(b_{n+3}^{\tau}\right)$ is a Galois extension of $k_{n}$. Since $\phi^{2}\left(b_{n+2}^{\tau}\right)=b_{n}$, we have $b_{n+2}^{\tau} \in\left\{b_{n+2},-\frac{1}{b_{n+2}}\right.$, $\left.\frac{b_{n+2}+1}{b_{n+2}-1},-\frac{b_{n+2}-1}{b_{n+2}+1}\right\}$ (see (2)). If $b_{n+2}^{\tau}$ is either $b_{n+2}$ or $-\frac{1}{b_{n+2}}$, then $b_{n+1}^{\tau}=\phi\left(b_{n+2}^{\tau}\right)=b_{n+1}$, which implies a contradiction that $k_{n+1} \subset k_{n}$. Therefore $b_{n+2}^{\tau}=$ $\frac{b_{n+2}+1}{b_{n+2}-1} \quad$ or $\quad b_{n+2}^{\tau}=-\frac{b_{n+2}-1}{b_{n+2}+1}$. Then $\left(b_{n+2}^{\tau}\right)^{2}-1=$ $\pm \frac{4 b_{n+2}}{\left(b_{n+2} \mp 1\right)^{2}}$. Since $\quad b_{n+3}^{\tau}=-i\left(b_{n+2}^{\tau} \pm \sqrt{\left(b_{n+2}^{\tau}\right)^{2}-1}\right)$, $k_{n+3}^{\tau} k_{n+3}^{\left(b_{n+3}+1\right)}=k_{n+3}\left(\sqrt{b_{n+2}}\right) \subset K_{n+3}$ by Lemma 2.4. Since $\left[K_{n+3}: k_{n+3}\right] \leq 2$, we have $K_{n+3}=k_{n+3}^{\tau} k_{n+3}$ if $\sqrt{b_{n+2}} \notin k_{n+3}$. Suppose that $\sqrt{b_{n+2}} \in k_{n+3}$. Since $k_{j}\left(\sqrt{b_{j-1} b_{j}}\right)=k_{j+1} \subset k_{n+3}$ for any $n+2 \geq j \geq 1$, we have $\sqrt{b_{j-1}} \in k_{n+3}$ if $\sqrt{b_{j}} \in k_{n+3}$, and hence $\sqrt{b_{0}} \in k_{n+3}$ by induction. Then $K_{n+3}=k_{n+3}=$ $k_{n+3}^{\tau} k_{n+3}$. Therefore $K_{n+3} / k_{n}$ is a Galois extension.

By Lemma 2.3, $k_{n+1}\left(\sqrt{b_{n+1}\left(b_{n+1}+1\right)}\right)$ is a subextension of $k_{n+3} / k_{n}$ of degree 4 . Since

$$
b_{n+1}=\frac{\left(\sqrt{b_{n+1}\left(b_{n+1}+1\right)}\right)^{2}-1}{1-2 b_{n} i},
$$

one can easily see that the minimal polynomial of $\sqrt{b_{n+1}\left(b_{n+1}+1\right)}$ over $k_{n}$ is

(3) $x^{4}+a_{n} x^{2}+c_{n}$

$$
=x^{4}+\left(4 b_{n}^{2}+2 b_{n} i-2\right) x^{2}+b_{n}(1+i)^{2} .
$$

Then

$$
\begin{aligned}
c_{n}\left(a_{n}^{2}-4 c_{n}\right) & =4 b_{n}\left(b_{n}^{2}-1\right)(1+i)^{2}\left(2 b_{n}+i\right)^{2} \\
& \equiv b_{n}\left(b_{n}^{2}-1\right) \bmod \left(k_{n}^{\times}\right)^{2} .
\end{aligned}
$$

Lemma 2.6. The following three statements are equivalent:
(a) $N_{0} \neq \emptyset$.
(b) $1 \in N_{0}$, i.e., $N_{0}$ is the set of all positive rational integers.
(c) $\sqrt{b_{0}\left(b_{0}^{2}-1\right)} \in k$.

Proof. (a) $\Rightarrow$ (b): Put $n_{0}=\min N_{0}$. Suppose that $n_{0} \geq 3$. Then $K_{n_{0}}=k_{n_{0}}$ which is a Galois extension of $k_{n_{0}-3}$ by Lemma 2.5. Since $\sqrt{b_{0}} \notin k_{n_{0}-1}$ and $\sqrt{b_{0}} \in k_{n_{0}}$, we have $k_{n_{0}-1} \cap K_{n_{0}-3}=k_{n_{0}-3}$ and $k_{n_{0}}=K_{n_{0}-1}$. Since $k_{n_{0}-1} / k_{n_{0}-3}$ is a $[2,2]$-extension, $k_{n_{0}} / k_{n_{0}-3}$ is a $[2,2,2]$-extension. In particular, $k_{n_{0}-2}\left(\sqrt{b_{n_{0}-2}\left(b_{n_{0}-2}+1\right)}\right) / k_{n_{0}-3}$ is a [2,2]-extension. By (3), we have $\sqrt{c_{n_{0}-3}} \in k_{n_{0}-3}$ (see e.g. [10, Corollary 2.2.4]), i.e., $\sqrt{b_{n_{0}-3}} \in k_{n_{0}-3}$. This contradicts the validity of $C\left(b_{n_{0}-3}\right)$ by Lemma 2.3 . Therefore $n_{0} \leq 2$ if $N_{0} \neq \emptyset$. Suppose that $n_{0}=2$. Then $\sqrt{b_{0}} \in k_{2}$, and hence $k\left(\sqrt{b_{0}}\right) / k$ is a quadratic subextension of the $[2,2]$-extension $k_{2} / k$. Since $\sqrt{b_{0}} \notin k_{1}$, i.e., $\quad k\left(\sqrt{b_{0}}\right) \neq k_{1}$, we have either $k\left(\sqrt{b_{0}}\right)=k\left(\sqrt{b_{0}\left(b_{0}+1\right)}\right) \quad$ or $\quad k\left(\sqrt{b_{0}}\right)=$ $k\left(\sqrt{b_{0}\left(b_{0}-1\right)}\right)$. Then $\sqrt{b_{0}+1} \in k$ or $\sqrt{b_{0}-1} \in k$. This contradicts to the condition $C\left(b_{0}\right)$. Thus we have $n_{0}=1 \in N_{0}$.

(b) $\Leftrightarrow(\mathrm{c})$ : We have $\sqrt{b_{0}} \in k_{1}$ if and only if $k\left(\sqrt{b_{0}}\right)=k\left(\sqrt{b_{0}^{2}-1}\right)$, i.e., $\sqrt{b_{0}\left(b_{0}^{2}-1\right)} \in k$.

By Lemma 2.6, we have $K_{1}=k_{1}=k_{0}\left(\sqrt{b_{0}}\right) \neq$ $k_{0}$ if $N_{0} \neq \emptyset$. Put

$$
K_{0}= \begin{cases}k_{0} & \text { if } N_{0} \neq \emptyset, \\ k_{0}\left(\sqrt{b_{0}}\right) & \text { if } N_{0}=\emptyset .\end{cases}
$$

Then $k_{m} \cap K_{n}=k_{n}$ and $K_{m}=k_{m} K_{n}$ for any $m \geq$ $n \geq 0$. By Lemma 2.3 , we obtain a diagram

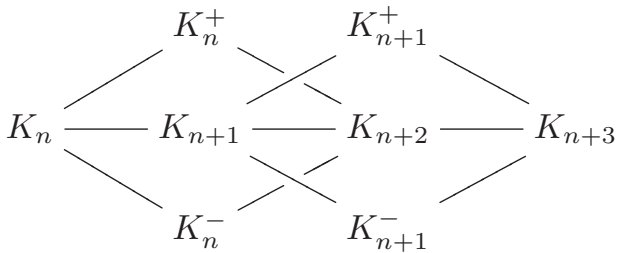

for all $n \geq 0$, where $K_{n}^{ \pm}=K_{n}\left(\sqrt{b_{n}\left(b_{n} \pm 1\right)}\right)$.

Lemma 2.7. The following two statements hold true:

- If $N_{0} \neq \emptyset$, then $k_{n+3} / k_{n}$ is a $[2,4]$-extension for each $n \geq 0$.

- If $N_{0}=\emptyset$, then $K_{n+3} / K_{n}$ is a $[2,4]$-extension for each $n \geq 1$, and

$$
\begin{aligned}
K_{3}=K_{0}\left(\sqrt{b_{0}\left(b_{0}-1\right)}, \sqrt{\left(\sqrt{b_{0}}+1\right)\left(\sqrt{b_{0}}-i\right)},\right. \\
\left.\sqrt{\left(\sqrt{b_{0}}-1\right)\left(\sqrt{b_{0}}+i\right)}\right) .
\end{aligned}
$$

Proof. By Lemmas 2.4 and 2.5, $K_{n+3} / K_{n}$ is a Galois extension of degree 8 for each $n \geq 0$. Since $\left[K_{n+1}^{+}: K_{n}\right]=4$, the polynomial (3) is irreducible over $K_{n}$. If $n \geq 1, K_{n}\left(\sqrt{b_{n}^{2}-1}\right)=K_{n+1}=K_{n}\left(\sqrt{b_{n}}\right)$ by Lemma 2.4 , which implies that $\sqrt{b_{n}} \notin K_{n}$ and 
$\sqrt{b_{n}\left(b_{n}^{2}-1\right)} \in K_{n}$. By Lemma 2.6, $\sqrt{b_{0}} \notin k=K_{0}$ and $\sqrt{b_{0}\left(b_{0}^{2}-1\right)} \in k_{0}=K_{0}$ if $N_{0} \neq \emptyset$. Hence $\sqrt{c_{n}}=$ $(1+i) \sqrt{b_{n}} \notin K_{n}$ and $\sqrt{c_{n}\left(a_{n}^{2}-4 c_{n}\right)} \in K_{n}$ for all $n \geq \delta$ (see (4)), where $\delta=0$ if $N_{0} \neq \emptyset$, and $\delta=1$ if $N_{0}=\emptyset$. Therefore $K_{n+1}^{+} / K_{n}$ is a cyclic extension of degree 4 (see e.g. [10, Corollary 2.2.4]) if $n \geq \delta$. This implies that $K_{n+3} / K_{n}$ is a [2,4]-extension for all $n \geq \delta$. In particular, we obtain the statement for $N_{0} \neq \emptyset$. Suppose that $N_{0}=\emptyset$. Then $x^{4}+a_{0} x^{2}+c_{0}$ is also the the minimal polynomial of $\sqrt{b_{1}\left(b_{1}+1\right)}$ over $K_{0}$. Recall that $b_{1}\left(b_{1}+1\right)=1-b_{1}\left(2 b_{0} i-1\right)$. Since $\sqrt{c_{0}}=(1+i) \sqrt{b_{0}} \in K_{0}, K_{1}^{+}=K_{0}\left(\sqrt{b_{1}\left(b_{1}+1\right)}\right)$ is a [2, 2]-extension of $K_{0}$ (see e.g. [10, Corollary 2.2.4]). Then the octic Galois extension $K_{3} / K_{0}$ contains two distinct $[2,2]$-extension $K_{1}^{+} / K_{0}$ and $K_{2} / K_{0}$, and hence $K_{3}=K_{0}^{-} K_{1}^{+}$is a $[2,2,2]$-extension of $K_{0}$. In fact, the four roots of $x^{4}+a_{0} x^{2}+c_{0}$ are

$$
\begin{aligned}
& \pm \frac{i}{2}\left(\sqrt{a_{0}+2 \sqrt{c_{0}}} \pm \sqrt{a_{0}-2 \sqrt{c_{0}}}\right) \\
& = \pm i\left(\left(\sqrt{b_{0}}+\frac{i-1}{2}\right) \sqrt{\left(\sqrt{b_{0}}+1\right)\left(\sqrt{b_{0}}-i\right)}\right. \\
& \left.\quad \pm\left(\sqrt{b_{0}}-\frac{i-1}{2}\right) \sqrt{\left(\sqrt{b_{0}}-1\right)\left(\sqrt{b_{0}}+i\right)}\right)
\end{aligned}
$$

and hence $K_{3}=K_{0}^{-}\left(\sqrt{a_{0} \pm 2 \sqrt{c_{0}}}\right)$. Thus we obtain the statement for $N_{0}=\emptyset$.

2.3. Number theoretic part. We shall consider the ramification and the class number parity. We denote by $\mathcal{O}_{F}$ the ring of integers in a number field $F$, and by $\mathcal{O}_{F}^{\times}$its unit group.

Lemma 2.8. If $b_{0} \in \mathcal{O}_{k}$, then;

- $k_{1} / k$ is unramified outside primes dividing $2\left(b_{0}^{2}-1\right)$.

- $k_{2} / k_{1}$ is unramified outside primes dividing $2 b_{0}$.

- $k_{n} / k_{2}$ is unramified outside 2 for any $n \geq 3$.

Proof. Recall that

$$
b_{n}^{2}+2 b_{n-1} i b_{n}-1=0
$$

for all $n \geq 1$. Then $b_{n} \in \mathcal{O}_{k_{n}}^{\times}$if $b_{n-1} \in \mathcal{O}_{k_{n-1}}$. Therefore $b_{n} \in \mathcal{O}_{k_{n}}^{\times}$for all $n \geq 1$ if $b_{0} \in \mathcal{O}_{k}$. Since

$$
k_{n+1}=k_{n}\left(\sqrt{b_{n}^{2}-1}\right)=k_{n}\left(\sqrt{b_{n-1} b_{n}}\right),
$$

we obtain the statements.

Lemma 2.9. Assume that $b_{0} \in \mathcal{O}_{k}$. Then the following two statements hold true:

- If $N_{0} \neq \emptyset$, then $k_{n} / k$ is unramified outside 2 for any $n \geq 0$.

- If $N_{0}=\emptyset$, then $K_{n} / K_{1}$ is unramified outside 2 for any $n \geq 1$, and $K_{1} / K_{0}$ is unramified outside primes dividing $2\left(b_{0}^{2}-1\right)$.
Proof. Since $K_{4} / K_{1}$ is a $[2,4]$-extension by Lemma $2.7, K_{2}^{+} / K_{1}$ is a cyclic extension of degree 4 , which is unramified outside $2 b_{0}$ by Lemma 2.8 . Since $K_{2}^{+} / K_{2}$ is unramified outside 2 by Lemma 2.8 , any prime $v$ not dividing 2 does not ramify in $K_{2}^{+} / K_{1}$. Hence $K_{2} / K_{1}$ is unramified outside 2 . By Lemma 2.8, $K_{n} / K_{1}$ is unramified outside 2 for any $n \geq 1$. If $N_{0}=\emptyset$, then $k_{1} \cap K_{0}=k$ and $K_{1}=k_{1} K_{0}$. Hence we obtain the statement for $N_{0}=\emptyset$. Suppose that $N_{0} \neq \emptyset$. Then $k_{1}=k\left(\sqrt{b_{0}^{2}-1}\right)=k\left(\sqrt{b_{0}}\right)$ by Lemma 2.6, and hence $k_{1} / k$ is unramified outside $2\left(b_{0}^{2}-1\right)$ and unramified outside $2 b_{0}$. Since $\mathcal{O}_{k}\left(b_{0}^{2}-1\right)+\mathcal{O}_{k} b_{0}=\mathcal{O}_{k}, k_{1} / k$ is unramified outside 2. Thus we obtain the statement for $N_{0} \neq \emptyset$.

We need the following result, which is a part of [17, Theorem 2.1] or [13, Proposition 1].

Proposition 2.10. Let $S$ be a finite set of primes of a subfield of a number field $K$, and let $T$ be a subset of $S$. Let $K^{\prime \prime} / K$ be a cyclic quartic extension, which is unramified outside $S$ and totally ramified at any primes lying over $S$. Let $K^{\prime} / K$ be the unique quadratic subextension of $K^{\prime \prime} / K$. If $2 \nmid\left|C l^{T}\left(K^{\prime}\right)\right|$, then $2 \nmid\left|C l^{T}\left(K^{\prime \prime}\right)\right|$.

Suppose that $b_{0} \in \mathcal{O}_{k}$. By Lemmas 2.7 and 2.9, $K_{n+3} / K_{n}$ is a $[2,4]$-extension unramified outside 2 for any $n \geq \delta$, where $\delta=0$ if $N_{0} \neq \emptyset$, and $\delta=1$ if $N_{0}=\emptyset$.

Lemma 2.11. Suppose that $n \geq \delta$. Assume that $K_{n+2} / K_{n}$ is totally ramified at any primes lying over 2 and $2 \nmid\left|C l^{T}\left(K_{n+2}\right)\right|$. Then $K_{n+3} / K_{n+1}$ is totally ramified at any primes lying over 2 and $2 \nmid\left|C l^{T}\left(K_{n+3}\right)\right|$.

Proof. Since $K_{n+2} / K_{n}^{+}$is ramified at any primes $v \mid 2$, the cyclic quartic extension $K_{n+3} / K_{n}^{+}$ is totally ramified at any $v \mid 2$. Hence $K_{n+3} / K_{n+1}$ is totally ramified at any primes lying over 2 . Since $2 \nmid\left|C l^{T}\left(K_{n+2}\right)\right|$, we have $2 \nmid\left|C l^{T}\left(K_{n+3}\right)\right|$ by Proposition 2.10 for the cyclic quartic extension $K_{n+3} / K_{n}^{+}$.

Suppose that $n \geq \delta$. By using Lemma 2.11 recursively, we see that $2 \nmid\left|C l^{T}\left(K_{m}\right)\right|$ for any $m \geq$ $n$ if $K_{n+2} / K_{n}$ is totally ramified at any primes lying over 2 and $2 \nmid\left|C l^{T}\left(K_{n+2}\right)\right|$. By combining Lemmas 2.6, 2.7, 2.9 and this fact, the proof of Theorem 1.1 is completed.

3. Examples. The following result by Iwasawa ([8]) is also useful to find examples.

Proposition 3.1. Let $K^{\prime} / K$ be a quadratic extension ramified at only one prime $v$. Put $T=\emptyset$ or $T=\{v\}$. If $2 \nmid\left|C l^{T}(K)\right|$, then $2 \nmid\left|C l^{T}\left(K^{\prime}\right)\right|$. 
Suppose that $\phi$ is defined as (1).

Example 3.2. Put $b_{0}= \pm i$, and put $k=$ $\mathbf{Q}(i)$. Since $b_{0}\left(b_{0}^{2}-1\right)= \pm(1+i)^{2}$, we have $\sqrt{b_{0}\left(b_{0}^{2}-1\right)} \in k$. Then

$$
k_{2}=\mathbf{Q}\left(i, b_{2}\right)=\mathbf{Q}\left(i, \sqrt{b_{0}}, \sqrt{b_{0}+1}\right)=\mathbf{Q}\left(\zeta_{8}, \sqrt{1+i}\right)
$$

is a [2,2]-extension of $k=\mathbf{Q}(i)$ which is unramified outside 2 and totally ramified at $1+i$. Hence the condition $C\left(b_{0}\right)$ is satisfied (see Remark 1.2). By Lemma $2.7, k_{n+3} / k_{n}$ is a $[2,4]$-extension unramified outside $1+i$ for any $n \geq 0$. By applying Proposition 3.1 for $k_{n} / k_{n-1}$ recursively, we see that $k_{\infty} / k$ is totally ramified at $1+i$, and that $2 \nmid\left|C l\left(k_{n}\right)\right|$ for any $n \geq 0$.

Remark 3.3. Note that

$$
-i \phi(i x)=\frac{i}{2}\left(x+\frac{1}{x}\right)=-(1+i)^{-2}\left(x+\frac{1}{x}\right)
$$

is a $\mathrm{PGL}_{2}$-conjugate of $\phi$. Put $\Phi(x)=i \phi(i x)$. Then $\Phi(x)$ is the $x$-coordinate of the endomorphism $[1+i]$ of the elliptic curve $E: y^{2}=x^{3}+x$ with complex multiplication by $\mathbf{Z}[i]$ and the $j$-invariant 1728 (see [16, p. 111, Proposition 2.3.1]). Since

$$
\begin{aligned}
\Phi^{n}(x) & =(-1)^{n}(-\Phi)^{n}(x)=(-1)^{n}(-i \phi i)^{n}(x) \\
& =(-1)^{n+1} i \phi^{n}(i x),
\end{aligned}
$$

we have $(-1)^{n+1} i \phi^{-n}\left(b_{0}\right)=\Phi^{-n}\left(i b_{0}\right)$, and hence $k\left(\phi^{-n}\left(b_{0}\right)\right)=k\left(\Phi^{-n}\left(i b_{0}\right)\right)$. Since $\Phi^{-3}(\infty)=\{ \pm 1, \pm i\}$ and $\Phi( \pm 1)= \pm i$, we have $\Phi^{-n-3}(\infty)=\Phi^{-n}( \pm 1) \cup$ $\Phi^{-n+1}( \pm 1)$. If $b_{0}=\mp i$ as in Example 3.2, then

$$
k\left(\phi^{-n}\left(b_{0}\right)\right)=k\left(\Phi^{-n}( \pm 1)\right)=k\left(\Phi^{-n-3}(\infty)\right)
$$

contains the ray class field $\mathbf{Q}(i)\left(\xi^{2} \mid \xi \in \Phi^{-n-3}(\infty)\right)$ of $\mathbf{Q}(i)$ modulo $(1+i)^{n+3}$ (see [16, p. 135, Theorem $5.6])$.

Example 3.4. Put $b_{0}= \pm i$, and put $k=$ $\mathbf{Q}(i, \sqrt{q})$ with an odd prime number $q$. By Proposition 3.1, the class number of $\mathbf{Q}\left(\sqrt{q^{*}}\right)$ is odd, where $q^{*}=(-1)^{\frac{q-1}{2}} q \equiv 1(\bmod 4)$. By Example 3.2 , the conditions $\sqrt{b_{0}\left(b_{0}^{2}-1\right)} \in k$ and $C\left(b_{0}\right)$ are satisfied (see Remark 1.2), and $k_{n+3} / k_{n}$ is a [2,4]-extension unramified outside 2 and totally ramified at any primes lying over 2 for any $n \geq 0$.

If $q^{*} \equiv 5(\bmod 8)$, then 2 does not split in $k / \mathbf{Q}$. By Proposition 3.1 for $k / \mathbf{Q}\left(\sqrt{q^{*}}\right)$, the class number of $k$ is odd. By applying Proposition 3.1 for $k_{n} / k_{n-1}$ recursively, we see that $2 \nmid\left|C l\left(k_{n}\right)\right|$ for any $n \geq 0$.

Put $T=\{2\}$. By using PARI/GP ([14]), one can see that $2 \nmid\left|C l^{T}\left(k_{2}\right)\right|$ if $q \equiv 7(\bmod 16)$ and $q<$

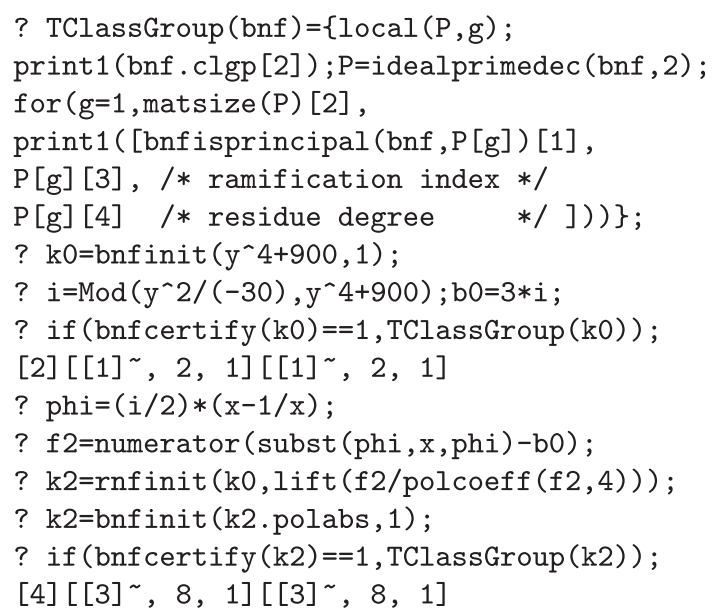

Fig. 1. PARI/GP for Example 3.5.

100 (i.e., $q \in\{7,23,71\}$ ). Then $k$ has two primes lying over 2. By Lemma 2.11, $2 \nmid\left|C l^{T}\left(k_{n}\right)\right|$ for any $n \geq 0$.

Example 3.5. Put $b_{0}=3 i$ and $k=$ $\mathbf{Q}\left(i, \sqrt{b_{0}\left(b_{0}^{2}-1\right)}\right)=\mathbf{Q}(\sqrt{-30 i})=\mathbf{Q}(i, \sqrt{15})$. Then we obtain the following results by using PARI/GP ([14]): The condition $C\left(b_{0}\right)$ is satisfied (see Remark $1.2)$, and the prime $1+i$ of $\mathbf{Q}(i)$ splits in $k / \mathbf{Q}(i)$. Moreover, $k_{2} / k$ is totally ramified at the two primes of $k$, and $2 \nmid\left|C l^{T}\left(k_{2}\right)\right|$ for $T=\{2\}$ (see Figure 1). By Lemma 2.11, $2 \nmid\left|C l^{T}\left(k_{n}\right)\right|$ for any $n \geq 0$.

On the other hand, suppose that $b_{0}=2-i$ and $k=\mathbf{Q}\left(i, \sqrt{b_{0}\left(b_{0}^{2}-1\right)}\right)=\mathbf{Q}(i, \sqrt{5})$. As seen in Example 3.4, the class number of $k$ is odd, and 2 does not split in $k / \mathbf{Q}$. Then $2 \nmid\left|C l\left(k_{n}\right)\right|$ for all $n \geq 0$, by Lemma 2.9 and the recursive use of Proposition 3.1 .

Example 3.6. Put $k=\mathbf{Q}(i)$. For $\quad b_{0} \in$ $\{3 i, 2-i, 1+4 i, 6-i, 3+2 i\}$, we obtain the following results by using PARI/GP ([14]): The condition $C\left(b_{0}\right)$ is satisfied and $\sqrt{b_{0}\left(b_{0}^{2}-1\right)} \notin k$.

If $b_{0}=3 i, K_{0} / \mathbf{Q}$ is totally ramified at 2 , and the unique prime of $K_{0}$ lying over 2 splits in $K_{1} / K_{0}$. Moreover, assuming GRH, $K_{3} / K_{1}$ is totally ramified at any primes lying over 2 , and $2 \nmid\left|C l^{T}\left(K_{3}\right)\right|$ for $T=\{2\}$. By Theorem 1.1, $2 \nmid\left|C l^{T}\left(K_{n}\right)\right|$ for any $n \geq 1$ under GRH.

If $b_{0} \in\{2-i, 1+4 i, 6-i\}, 2$ does not split in $K_{1} / \mathbf{Q}$ and $\left|C l\left(K_{1}\right)\right|=1$. If $b_{0}=3+2 i$, then 2 does not split in $K_{2} / \mathbf{Q},\left|C l\left(K_{1}\right)\right|=2$ and $\left|C l\left(K_{2}\right)\right|=1$. By Lemma 2.9 and the recursive use of Proposi- 
tion 3.1, we see that the class number of $K_{n}$ is odd for any $n \geq 2$.

Remark 3.7. We have not yet found any example of $b_{0}$ and $k$ such that $2 \nmid\left|C l\left(K_{n}\right)\right|$ and $K_{n}$ has at least two ramified primes lying over 2 for all sufficiently large $n \geq \delta$.

Acknowledgements. The authors thank the referees for helpful comments for the improvement of this paper. This work was supported by JSPS KAKENHI Grant Number JP17K05167.

\section{References}

[ 1 ] W. Aitken, F. Hajir and C. Maire, Finitely ramified iterated extensions, Int. Math. Res. Not. 2005, no. 14, 855-880.

[ 2 ] N. Boston and R. Jones, The image of an arboreal Galois representation, Pure Appl. Math. Q. 5 (2009), no. 1, 213-225.

[ 3 ] M. R. Bush, W. Hindes and N. R. Looper, Galois groups of iterates of some unicritical polynomials, Acta Arith. 181 (2017), no. 1, 57-73.

[ 4 ] L. Caputo and F. A. E. Nuccio Mortarino Majno di Capriglio, On fake $\mathbf{Z}_{p}$-extensions of number fields, arXiv:0807.1135.

[ 5 ] J. Cullinan and F. Hajir, Ramification in iterated towers for rational functions, Manuscripta Math. 137 (2012), no. 3-4, 273-286.

[6] A. A. Cuoco and P. Monsky, Class numbers in $\mathbf{Z}_{p}^{d}$-extensions, Math. Ann. 255 (1981), no. 2, $235-258$.

[ 7 ] R. Greenberg, On the Iwasawa invariants of totally real number fields, Amer. J. Math. 98
(1976), no. 1, 263-284.

[ 8 ] K. Iwasawa, A note on class numbers of algebraic number fields, Abh. Math. Sem. Univ. Hamburg 20 (1956), 257-258.

[ 9 ] K. Iwasawa, On $\Gamma$-extensions of algebraic number fields, Bull. Amer. Math. Soc. 65 (1959), 183226.

[10 ] C. U. Jensen, A. Ledet and N. Yui, Generic polynomials: Constructive Aspects of the Inverse Galois Problem, Mathematical Sciences Research Institute Publications, 45, Cambridge University Press, Cambridge, 2002.

[11] T. Kataoka, An Iwasawa theory for non-Galois extension fields, RIMS Kôkyûroku 658 (1988), 34-42 (in Japanese).

[ 12 A. Lei, Estimating class numbers over metabelian extensions, Acta Arith. 180 (2017), no. 4, 347364.

[13 ] Y. Mizusawa and K. Yamamoto, On 2-adic Lie iterated extensions of number fields arising from a Joukowski map. (to appaer in Tokyo J. Math.).

[ 14 ] The PARI Group, PARI/GP version 2.7.4, Univ. Bordeaux, 2015. http://pari.math.u-bordeaux. fr/

[ 15 ] G. Perbet, Sur les invariants d'Iwasawa dans les extensions de Lie $p$-adiques, Algebra Number Theory 5 (2011), no. 6, 819-848.

[16 ] J. H. Silverman, Advanced topics in the arithmetic of elliptic curves, Graduate Texts in Mathematics, 151, Springer-Verlag, New York, 1994.

[ 17 K K. Yamamoto, On iterated extensions of number fields arising from quadratic polynomial maps, J. Number Theory 209 (2020), 289-311. 